\title{
Broadband Phase-Sensitive Single InP Nanowire Photoconductive Terahertz Detectors
}

\author{
Kun Peng, ${ }^{* \dagger}{ }^{\dagger}$ Patrick Parkinson, ${ }^{\ddagger}$ Jessica L. Boland, ${ }^{\S}$ Qian Gao, ${ }^{\dagger}$ Yesaya C. Wenas, ${ }^{\dagger}$
} Christopher L. Davies, ${ }^{\S}$ Ziyuan Li, ${ }^{\dagger}$ Lan Fu, ${ }^{*}{ }^{\dagger}$ Michael B. Johnston, ${ }^{\S}$ Hark H. Tan, ${ }^{\dagger}$ and Chennupati Jagadish ${ }^{\dagger}$

${ }^{\dagger}$ Department of Electronic Materials Engineering, Research School of Physics and Engineering, The Australian National University, Canberra, ACT 2601, Australia

${ }^{\ddagger}$ School of Physics and Astronomy and the Photon Science Institute, University of Manchester, M13 9PL, Manchester, United Kingdom

${ }^{\S}$ Department of Physics, University of Oxford, Clarendon Laboratory, Parks Road, Oxford, OX1 3PU, United Kingdom

\section{Supporting Information}

ABSTRACT: Terahertz time-domain spectroscopy (THz-TDS) has emerged as a powerful tool for materials characterization and imaging. A trend toward size reduction, higher component integration, and performance improvement for advanced THz-TDS systems is of increasing interest. The use of single semiconducting nanowires for terahertz $(\mathrm{THz})$ detection is a nascent field that has great potential to realize future highly integrated $\mathrm{THz}$ systems. In order to develop such components, optimized material optoelectronic properties and careful device design are necessary. Here, we present antenna-optimized photoconductive detectors based on single InP nanowires with superior properties of high carrier mobility $\left(\sim 1260 \mathrm{~cm}^{2} \mathrm{~V}^{-1} \mathrm{~s}^{-1}\right)$ and low dark current $(\sim 10 \mathrm{pA})$, which exhibit excellent sensitivity and broadband performance. We demonstrate that these nanowire $\mathrm{THz}$ detectors can provide high quality time-domain spectra for materials characterization in a THz-TDS system, a critical step toward future application in advanced THz-TDS system with high spectral and spatial resolution.

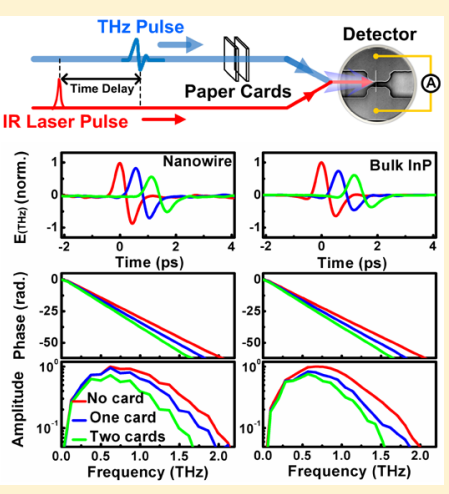

KEYWORDS: Nanowire, terahertz optoelectronics, ultrafast, nano-optics

$\mathrm{T}$ erahertz time-domain spectroscopy (THz-TDS $)^{1,2}$ has attracted enormous attention over the last two decades for a wide range of scientific, industrial, medical, and military applications. ${ }^{3-5}$ It has shown particular promise for applications in materials science, due to its ability to detect both the amplitude and the phase of the $\mathrm{THz}$ radiation interacting with a material. It can thereby provide information on the physical properties of a material represented by the absorption and refractive index in the far-infrared region. In common research and commercial THzTDS systems, photoconductive (Auston) switches ${ }^{6}$ based on semiconductor materials, such as low-temperature grown GaAs, ${ }^{7}$ ion-damaged silicon-on-sapphire ${ }^{8}$ or $\mathrm{InP}^{9}{ }^{9}$ and ErAs:(In)$\mathrm{GaAs},{ }^{10,11}$ have been widely used for $\mathrm{THz}$ detection owing to their low cost (compared to electro-optic crystals ${ }^{12}$ ) and simple operating principle. However, semiconductors suitable for $\mathrm{THz}$ detection typically require both an ultrashort charge carrier lifetime and reasonable carrier mobility, which need a compromise to be reached to ensure a sufficient response level while minimizing current noise. ${ }^{13}$ To achieve these properties in bulk semiconductors, low-temperature molecular beam epi$\operatorname{taxy}^{7,14}$ and/or postgrowth processing steps, such as ionimplantation $^{9,15}$ and annealing, are required. The low yields and challenges in reproducibility of low temperature growth as well as complicated multiple-step fabrication procedures give researchers a further motivation to explore new materials for $\mathrm{THz}$ detection. III-V semiconductor nanowires have been considered as ideal alternatives to their bulk/planar counterparts for use as active components in THz systems. ${ }^{16-18}$ They offer many desirable material properties: a direct and tunable band gap, good carrier mobility close to that of bulk materials, ${ }^{19}$ and short carrier lifetime on the picosecond to nanosecond time scale, ${ }^{19,20}$ which can be adjusted by controlling the crystal quality $^{21}$ or surface state density. ${ }^{20}$ Therefore, using III-V nanowires for $\mathrm{THz}$ detection could avoid additional material processing steps after crystal growth, as well as the high yield of III-V nanowires grown on a standard wafer suggests a possible high yield of nanowire-based devices, advantageous in both reduced cost and complexity. Furthermore, due to their onedimensional structure and nanoscale size, nanowires exhibit great promise for highly integrated $\mathrm{THz}$ systems, for example, as subwavelength detector elements for near-field imaging ${ }^{22}$ or as detecting components for "on-chip" $\mathrm{THz}$ spectrometers. ${ }^{23,24}$

To date, InAs, InAs/InSb nanowires (as plasma-wave detectors based on nanowire field-effect transistors for

Received: April 13, 2016

Revised: June 30, 2016

Published: July 14, 2016 
imaging $^{16,17,25}$ ) and GaAs/AlGaAs core-shell nanowires (as photoconductive sensors for spectroscopy ${ }^{18}$ ) have been reported for $\mathrm{THz}$ detection. However, in these studies the nanowire-based devices are still at an early stage of development with many fundamental issues and functionalities yet to be understood and explored for further implementation in industrial THz-TDS environment. Compared with other III-V nanowires, ${ }^{18,26}$ InP nanowires have shown high photosensitivity ${ }^{27-29}$ and radiative quantum efficiency ${ }^{27}$ due to suppressed nonradiative recombination originating from their intrinsically low surface recombination velocity. ${ }^{1,30}$ This eliminates the need for additional chemical or heterostructural surface passivation to enhance radiative recombination and prevent oxidative degradation, ${ }^{31,29}$ which are otherwise essential for the (InAl)GaAs material system in device applications. Therefore, growing high crystal quality InP nanowires has been a significant topic of interest ${ }^{27,32-35}$ for many applications, and the understanding and development in their growth mechanisms and techniques have led to successful demonstrations of both highly efficient solar cells ${ }^{35}$ and roomtemperature nanolasers. ${ }^{27}$

In this work, we demonstrate single-InP-nanowire photoconductive antennas (SNW-PCAs) exhibiting remarkable performance for broadband $\mathrm{THz}$ detection. The highly sensitive broadband $\mathrm{THz}$ detectors are developed from single stackingfault-free InP nanowires grown by selective-area (SA) metalorganic vapor phase epitaxy (MOVPE) with an optimized antenna design. By further employing these SNW-PCAs as microscopic coherent sensors in a THz-TDS system, the $\mathrm{THz}$ transmission spectra of simple objects were successfully measured showing excellent spectral and phase sensitivities, a critical step to demonstrate the promise of SNW-PCAs for future THz-TDS applications.

InP nanowires were grown onto bulk InP substrates using the SA-MOVPE technique within a commercial MOVPE reactor. The recipes used followed a previously published approach; ${ }^{27,36}$ more details are in the Supporting Information. Pure wurtzite, structurally uniform and high radiative quantum efficiency ${ }^{27,36}$ InP nanowires were produced with a range of diameters from 240 to $260 \mathrm{~nm}$ and lengths from 8 to $11 \mu \mathrm{m}$ (see Figure S1). After growth, the InP nanowires were mechanically dispersed on z-cut quartz substrates, which are transparent to $\mathrm{THz}$ radiation. Conventional UV photolithography was then employed to pattern the electrodes onto the nanowires. An oxygen plasma etch was used for further removal of the photoresist residue on nanowires, followed by a $9.3 \% \mathrm{HCl}$ chemical etching to remove native oxide layer formed on the nanowire surface. Finally, the detector structures were metallized using electron beam evaporation, followed by lift-off to form $\mathrm{Ti} / \mathrm{Au}(10 \mathrm{~nm} / 300$ $\mathrm{nm})$ contacts. Bow-tie ${ }^{37}$ and strip-line ${ }^{38}$ antenna elements, which have been optimized and simulated using finite-difference timedomain (FDTD) simulations (details are described in Supporting Information, Figure S2), were chosen as electrode geometries for our $\mathrm{InP}$ nanowire detectors and have been proven to provide a broadband $\mathrm{THz}$ response in bulk detectors. ${ }^{37}$ Figure 1 shows typical images of a single InP nanowire and geometry of the InP SNW-PCAs used in this work.

For signal-to-noise optimized $\mathrm{THz}$ detection, semiconductor materials with ultrafast (subpicosecond) photoconductivity rise times, short (picosecond to nanosecond) charge recombination lifetimes, high carrier mobilities, and large dark resistivities are desired. ${ }^{39}$ The photoconductivity rise time determines the speed and detection bandwidth of the device. ${ }^{13}$ The recombination lifetime determines the detector operation type and noise level,

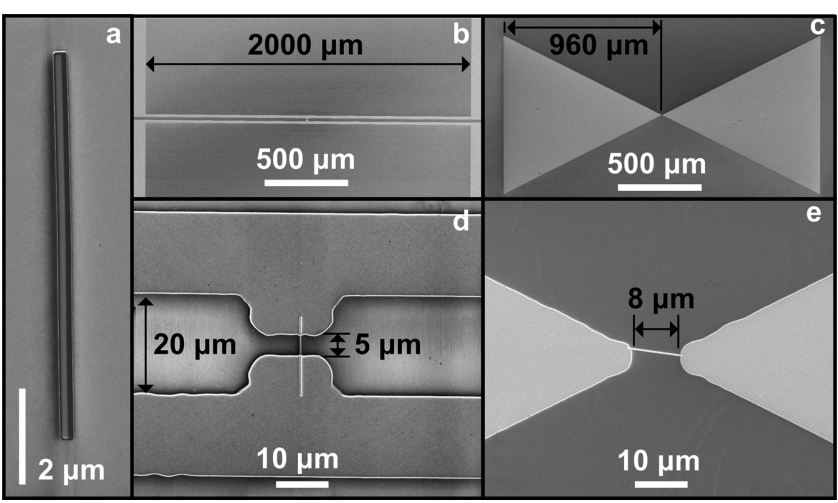

Figure 1. Representative scanning-electron-microscope images of a single InP nanowire and InP SNW-PCAs used in this study, labeled with dimensions: (a) a single InP nanowire; ( $b$ and $d$ ) a nanowire detector with strip-line geometry; (c and e) a nanowire detector with bow-tie geometry. (Top row) Overviews of the fabricated detectors. (Bottom row) Close-up images of the central area of the detectors.

while the mobility and resistivity contribute to the detector response level and sensitivity. ${ }^{13}$ Thus, we first evaluate the photoconductivity rise time, carrier lifetime and mobility of our InP nanowires by using time-domain optical pump-THz probe spectroscopy. ${ }^{18,40}$ A monoexponential carrier decay was observed for an ensemble of InP nanowires with a subpicosecond photoconductivity rise time ${ }^{30}$ and a photoconductivity lifetime of $\sim 1.71$ ns as shown in Figure 2a, indicating that our InP SNWPCAs are integrating detectors ${ }^{13,18}$ (which determines the signal processing technique required to recover the $\mathrm{THz}$ electric field from the measured photocurrent ${ }^{18}$ ) with ultrafast switch-on speed. The mobility of InP nanowires was extracted by fitting the photoconductivity spectra and was found to be $1260 \pm 320 \mathrm{~cm}^{2}$ $\mathrm{V}^{-1} \mathrm{~s}^{-1}$ which is a factor of $\sim 2$ times higher than that of previously reported InP nanowires grown by the vapor-liquidsolid technique, ${ }^{19,30}$ indicating the high quality of the nanowires. Measurements of room-temperature direct-current photocurrents on the fabricated InP SNW-PCAs were then carried out. The photocurrents from InP SNW-PCAs were obtained under illumination with a pulsed $522 \mathrm{~nm}$ laser at a fluence of $0.8 \mathrm{~mJ} /$ $\mathrm{cm}^{2} /$ pulse and were found to be $30 \mathrm{nA}$ at $2 \mathrm{~V}$ with the dark currents at sub-nA level $(\sim 10 \mathrm{pA})$, indicating the low dark conductivity but high photosensitivity of our InP SNW-PCAs, which is desirable for low-noise photoconductive detection (see Figure S3 in Supporting Information).

Next, the SNW-PCAs were incorporated into a pulsed THzTDS spectrometer system ${ }^{18}$ for characterization as $\mathrm{THz}$ detectors. A schematic diagram of the THz-TDS system and details of the optical arrangement for this system are demonstrated in the Supporting Information (see Figure S4). Briefly, a femtosecond Ti:sapphire laser was used for $\mathrm{THz}$ generation and detection, which produces pulses at a center wavelength of $800 \mathrm{~nm}$ with a duration of $\sim 35 \mathrm{fs}$ and a repetition rate of $84.5 \mathrm{MHz}$. The pulsed laser was split into two beams. One beam (with a power of $\sim 136.2 \mathrm{~mW}$ ) was used to excite a conventional photoconductive GaAs THz emitter ${ }^{18}$ that is biased with a square wave of $\pm 400 \mathrm{~V}$ amplitude at $17 \mathrm{kHz}$. The other beam was used to excite the detector sample at a fluence of 1.9 $\mathrm{nJ} / \mathrm{cm}^{2} /$ pulse. The generated $\mathrm{THz}$ beam and excitation beam were aligned and spatially overlapped at the center area of the detector. A delay stage was used to change the time delay between $\mathrm{THz}$ and excitation beams, by which the generated $\mathrm{THz}$ signals were measured in the time domain. Figure $2 \mathrm{~b}$ shows a 

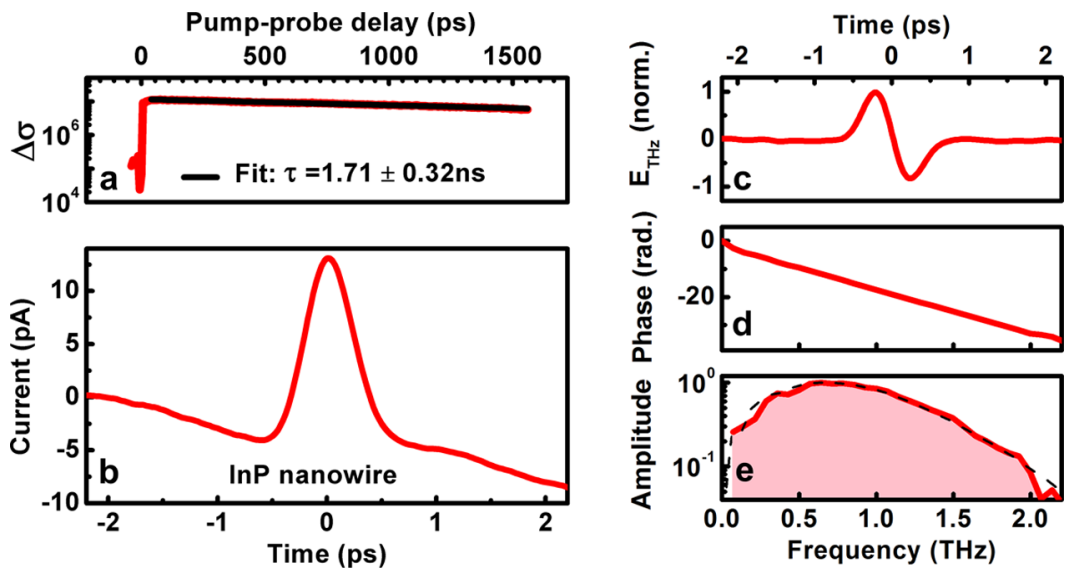

Figure 2. THz response characteristics of a typical InP SNW-PCA THz detector: (a) The decay of conductivity lifetime of InP nanowires (indicating the fabricated SNW-PCAs should be of integrating type); (b) THz induced current (measured from a single nanowire detector with strip-line electrode geometry); (c, d, e) processed data from b: calculated time-domain $\mathrm{THz}$ electrical field, phase, and amplitude frequency spectrum. The dashed line is the reference spectrum obtained from a standard bulk electro-optic crystal detector.

typical THz-induced transient photocurrent measured from our InP SNW-PCAs (data from various SNW-PCAs are given in the Supporting Information, Figure S5). Using the temporal current profile in Figure $2 b$, key information such as the waveform of incident $\mathrm{THz}$ electric field as a function of time, and the corresponding phase and amplitude spectrum, were extracted and are presented in Figure 2c, d, and e (using the calculation methods described in ref 18). It can be seen from Figure 2e that the $\mathrm{THz}$ response measured from our InP SNW-PCAs exhibit excellent signal-to-noise ratio (SNR) with a broad, usable detection bandwidth ranging from 0.1 to $2.0 \mathrm{THz}$. The measurement bandwidth of the photoconductive detector is determined by the following four factors: the duration of the excitation laser pulse, the antenna geometry, the $\mathrm{THz}$ source to be measured, and the properties of the detection material. The laser pulse duration, $\mathrm{THz}$ source, and antenna are the most important, while the material properties affect usable bandwidth through determining the signal-to-noise ratio. ${ }^{13}$ In our case, the measured bandwidth is limited by the bandwidth of the $\mathrm{THz}$ source $(<3 \mathrm{THz})$.

Traditional InP receivers (bulk $\mathrm{Fe}^{+}$-implanted $\mathrm{InP}^{9}$ ) based on the same device geometry designs (bow-tie and strip-line) were fabricated and characterized in the THz-TDS system as references for comparison. FDTD simulation results are also demonstrated together as we have found that the detector response strongly depends on the device geometry. Figure 3 shows the $\mathrm{THz}$ response obtained from our InP SNW-PCAs, simulated antennas, and ion-implanted InP receivers (bulk reference) for both the strip-line and bow-tie configurations. It can be seen that the response waveform and spectral profile measured from our InP SNW-PCAs agree perfectly with the simulation results and that good SNR was obtained for both bowtie and strip-line configurations. The bulk references show similar performances to those of nanowire detectors with a small distortion observed in the measured waveform signal and frequency spectrum, particularly for the case of the bulk bowtie detector. This may be attributed to the ion-implanted InP detector being of intermediate type ${ }^{13}$ (rather than integrating type) as determined by their relatively shorter carrier lifetime $(<6$ ps), which requires further correction in terms of deconvolution $^{13}$ (the correction method is described in Supporting Information). However, such correction will not affect the signal-to-noise analysis. Moreover, the traditional ion-implanted
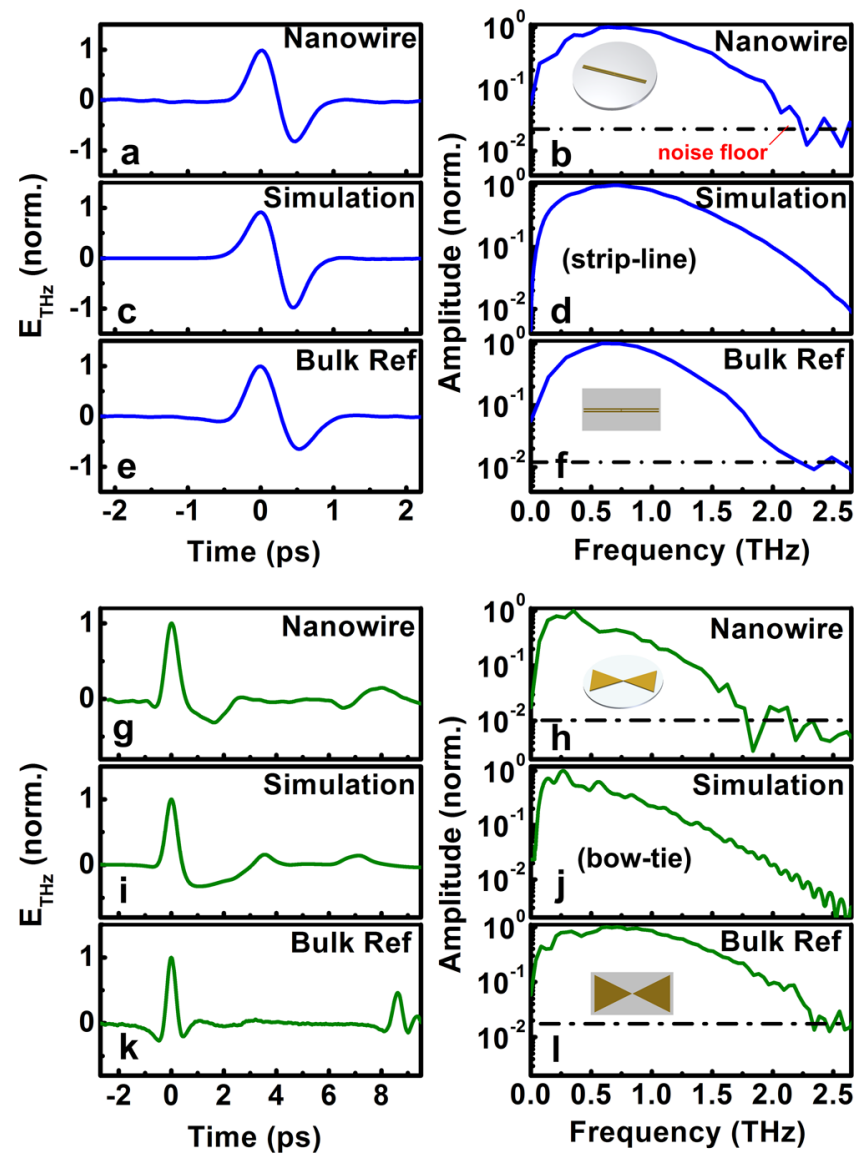

Figure 3. THz response obtained from (a, b, g, h) InP SNW-PCAs; (c, d, $i, j)$ simulated antennas; (e, f, k, l) bulk InP receivers, with contact geometry of strip lines (blue solid line) and bow tie (green solid line). All of the figures on the left are the time-domain electric field, and those on the right are their corresponding frequency-domain amplitude spectra. (Dash-dot line: noise floor. Inset: schematic diagrams of the samples.)

InP receiver shows a small replica of the main $\mathrm{THz}$ pulse at $\sim 8.5$ ps (see Figure 3k), due to a reflected $\mathrm{THz}$ pulse from the bottom surface of the bulk ion-implanted InP substrate $(\sim 0.4 \mathrm{~mm})$. In contrast, no such back reflection signal can be observed in the SNW-PCA spectra (see Figure 3g), since the SNW-PCAs are 
fabricated on thick quartz substrates $(\sim 2 \mathrm{~mm})$ with nanoscale active area (the definition of device active area can be found in Supporting Information), allowing longer temporal scan lengths for waveform measurements in the time domain to achieve higher resolution in the frequency spectrum. Indeed there is no intrinsic limit to the spectral resolution of the single nanowire detector if a supporting substrate is chosen such that the FabryPerot effects are removed (e.g., by choice of substrate geometry or refractive index).

To qualitatively investigate our SNW-PCAs, parametrized device performances of SNW-PCAs and traditional ionimplanted receivers are listed in Table 1. Details of the signal-

Table 1. Comparison of Device Performance between InP SNW-PCAs and Traditional Ion-Implanted InP Photoconductive Receivers $^{a}$

\begin{tabular}{|c|c|c|c|c|}
\hline \multirow{3}{*}{$\begin{array}{l}\text { detector type } \\
\text { performance }\end{array}$} & \multicolumn{4}{|c|}{ Photoconductive Antenna } \\
\hline & \multicolumn{2}{|c|}{ InP single nanowire } & \multicolumn{2}{|c|}{ ion-implanted InP bulk } \\
\hline & strip-line & bow-tie & strip-line & bow-tie \\
\hline dark current (at $1 \mathrm{~V}$ ) & $\sim 10 \mathrm{pA}$ & $\sim 10 \mathrm{pA}$ & $\sim 69200 \mathrm{pA}$ & $\sim 45700 \mathrm{pA}$ \\
\hline THz signal level & $19.5 \mathrm{pA}$ & $52.6 \mathrm{pA}$ & $676.6 \mathrm{pA}$ & $544.3 \mathrm{pA}$ \\
\hline SNR & 21 & 40 & 70 & 43 \\
\hline DR & 103 & 575 & 540 & 280 \\
\hline $\begin{array}{l}(-3 \mathrm{~dB}) \text { bandwidth } \\
(\mathrm{THz})\end{array}$ & 0.80 & 0.26 & 0.66 & 0.90 \\
\hline
\end{tabular}

${ }^{a}$ For SNR, signal is defined as the peak-to-peak current over one timedomain scan, and the standard deviation of the difference of two consecutive scans with identical parameters is defined as the noise. The dynamic range (DR) of $\mathrm{THz}$ time-domain data is the peak-topeak current to the standard deviation of the noise signal in the absence of $\mathrm{THz}$. to-noise calculations are provided in the Supporting Information. For traditional (bulk) photoconductive receivers, the main sources of electrical noise are Johnson-Nyquist and shot noise. The low dark current and high resistance of SNW-PCAs lead to very low Johnson-Nyquist noise; ${ }^{9}$ thus, noise in these nanowire devices is dominated by electrical shot noise and external sources of noise such as intensity fluctuations in the excitation laser. Considering the huge difference in detection material volume, our InP SNW-PCAs perform very competitively. The nanosize active area together with an insulating substrate of the SNWPCAs largely reduced the background noise (dark current and unintended photoinduced current), when compared to a conventional bulk $\mathrm{THz}$ receiver. In the latter case, unwanted current can arise from unintended photoexcitation of regions beyond the gap area. The present nanowire detector architecture is therefore able to improve the device signal-to-noise performance. This explains why SNW-PCAs show lower noise compared with bulk ion-implanted InP receivers, despite the former having a longer carrier lifetime. While the dynamic range for each detector is of comparable magnitude, significant differences remain, likely arising from different coupling effects between antenna geometry (bow-tie vs strip-line) and material parameters (nanowire vs bulk).

To demonstrate the potential of our InP SNW-PCAs for practical use, a number of $0.33( \pm 0.01) \mathrm{mm}$ paper cards were inserted into the $\mathrm{THz}$ path, and their transmission spectra were characterized using the InP SNW-PCAs. Figure 4a shows the optical arrangement used in this measurement. Figure $4 \mathrm{~b}-\mathrm{j}$ shows $\mathrm{THz}$ responses measured using an InP SNW-PCA, a bulk InP receiver, and a standard electro-optic detection crystal (ZnTe), with and without cards present in the THz-TDS system. It can be seen that, despite the nanoscale dimensions with $\sim \mathrm{pA}$ level $\mathrm{THz}$ photocurrent signals (raw data can be seen in
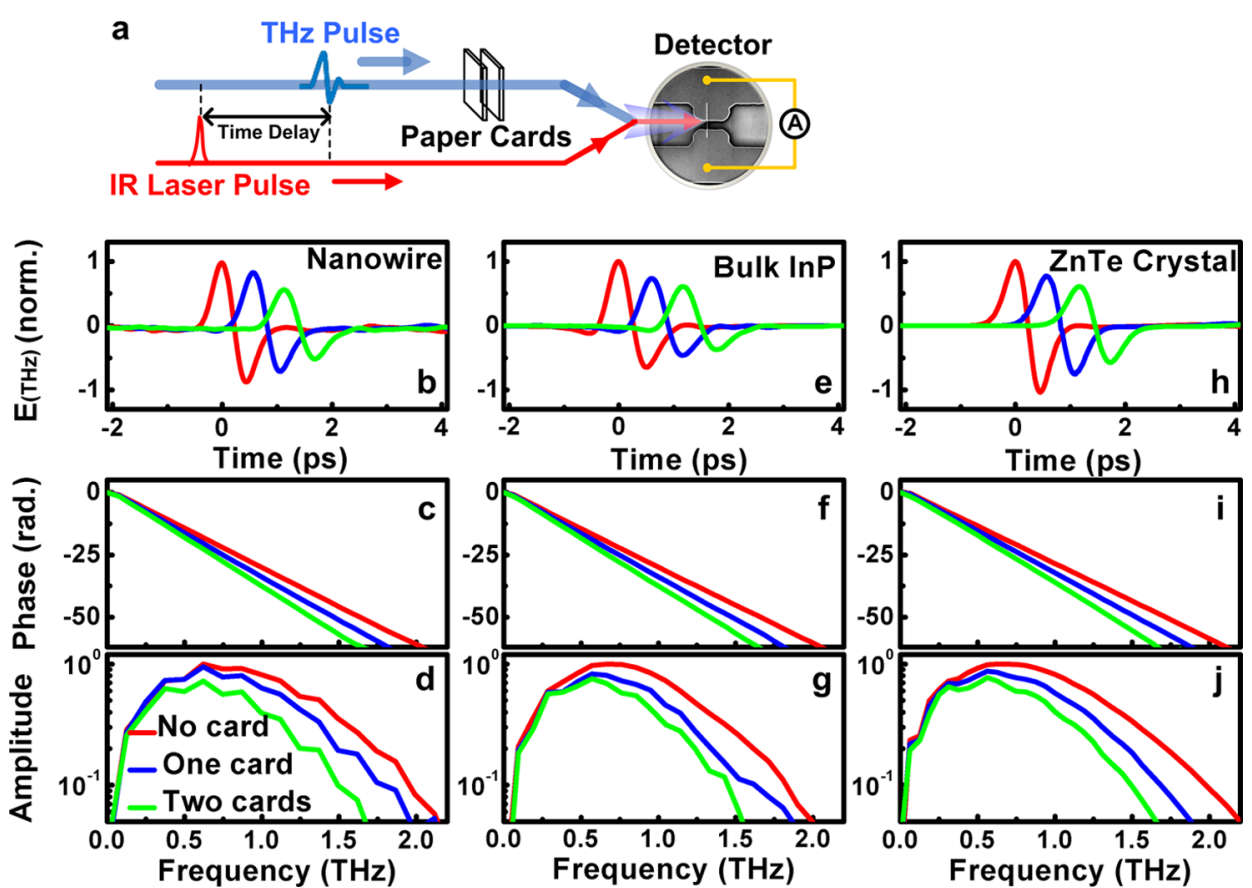

Figure 4. (a) Schematic representation of the transmission measurement in a THz-TDS system. Varying the time delay between excitation laser pulse and $\mathrm{THz}$ pulse allows profiling the electrical field of $\mathrm{THz}$ signal. $(\mathrm{b}-\mathrm{j}) \mathrm{THz}$ responses measured from three different types of detectors with and without paper cards present (red solid line: no card; blue solid line: one card; green solid line: two cards); (b, c, d) a strip-line InP SNW-PCA; (e, f, g) a strip-line ion-implanted InP receiver; $(h, i, j)$ a ZnTe electro-optic crystal. (Top row) Calculated time-domain electric field. (Center row) Frequency-domain phase information. (Bottom row) Frequency-domain amplitude spectrum. 

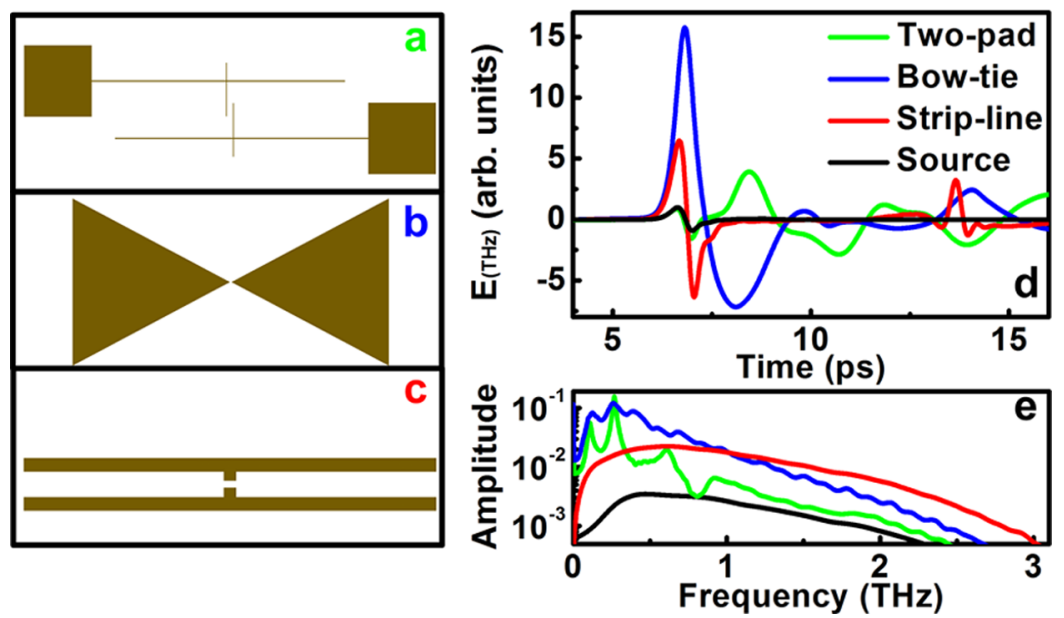

Figure 5. Schematic representations of device geometry studied by using FDTD simulations: (a) two-pad; (b) bow-tie; (c) strip-line. (d) Time-domain $\mathrm{THz}$ electrical field transient obtained from simulations with different antenna geometries. (e) Frequency spectrum of THz electrical field transient from d.

Supporting Information), our SNW-PCA shows comparable sensitivity and SNR to those of traditional bulk detectors in both measured $\mathrm{THz}$ amplitude and phase spectra. Based on the data shown in Figure 4, the absorption coefficient ${ }^{41}$ and refractive index spectra ${ }^{42-44}$ of the card were obtained (shown in Supporting Information). The refractive index of the paper card measured from the three different types of detectors is similar and nearly a constant $(\sim 1.55)$ over the frequency band extending from 0.4 to $1.6 \mathrm{THz}$ (see Figure S7 in Supporting Information), consistent with the value reported in ref 44 . In addition, the transmission spectrum of a low-pass filter and the water vapor absorption spectrum were also successfully characterized using the InP SNW-PCAs, confirming the functionality and practical value of the single nanowire $\mathrm{THz}$ detectors for absorption measurements (details are provided in Supporting Information).

It is well-known that the detector bandwidth response is a strong function of the resonant characteristic of the device geometry. ${ }^{18}$ To obtain some guidance for future work and estimate how the device design can be modified for tailoring the response performance and bandwidth of the detector, FDTD simulation results of three $\mathrm{THz}$ electrode geometries, including previously reported two-pad, ${ }^{18}$ bow-tie, and strip-line structures, were compared in Figure 5. More details of simulations are in the Supporting Information. Figure $5 \mathrm{~d}$ and e indicate that the twopad electrode design ${ }^{18}$ should strengthen the incident $\mathrm{THz}$ electrical field signal but cause a distortion in the measured waveform resulting in strong low-frequency resonances. The bow-tie electrode should also strengthen the incident $\mathrm{THz}$ signal by a larger factor, with a minor distortion in measured waveform as a result of relatively broadband response. The strip-line structure may be the best choice of geometry ${ }^{45}$ for $\mathrm{THz}$ detection while minimizing distortion of the incident radiation. All simulation results shown here are consistent with our experimental results obtained for the single-nanowire $\mathrm{THz}$ detectors. In the future, a careful design of device geometry optimized by modeling (such as a hybrid structure ${ }^{46}$ with a stripline-based spiral antenna for broadband operation), may further improve device performance, as well as strengthen the functional versatility of these InP SNW-PCAs.

In summary, we have demonstrated single nanowire photoconductive $\mathrm{THz}$ detectors, based on stacking-fault-free pure $\mathrm{WZ}$
InP nanowires, grown by SA-MOVPE. The InP nanowires exhibit high crystal quality as well as high carrier mobility, which is desirable for $\mathrm{THz}$ detection. By using FDTD simulations to optimize the device geometry design, a broad detection bandwidth $(0.1-2.0 \mathrm{THz})$, high amplitude, and phase sensitivity were achieved, which were found to be comparable to that of the traditional ion-implanted bulk $\mathrm{InP}$ receiver and standard electrooptic ZnTe crystal $\mathrm{THz}$ detector. The low-noise nature and the long time-domain sampling window (enabling higher spectral resolution measurements) benefit from the small active area and insulating substrates of these nanowires detectors. $\mathrm{THz}$ transmission measurements were performed in order to characterize the system and further demonstrate the prototype application of these InP SNW-PCAs in a THz-TDS system. These findings, along with advantages offered by the nanoscale device size and high spatial resolution, signify the great potential of these InP SNW-PCAs for future application in advanced compact $\mathrm{THz}$ systems.

\section{ASSOCIATED CONTENT}

\section{Supporting Information}

The Supporting Information is available free of charge on the ACS Publications website at DOI: 10.1021/acs.nanolett.6b01528.

Details of nanowire growth, dimensions of detector geometries used in FDTD simulations, photocurrent measurements, $\mathrm{THz}$ responses of InP SNW-PCAs, THzTDS system layout, definition of device active area, SNR calculations, deconvolution calculation, spectra of the absorption coefficient and refractive index of a paper card, absorption measurements by use of the InP SNW-PCA (PDF)

\section{AUTHOR INFORMATION}

\section{Corresponding Authors}

*E-mail: lan.fu@anu.edu.au (L.F.).

*E-mail: kun.peng@anu.edu.au (K.P.).

\section{Author Contributions}

P.P., L.F., H.H.T., C.J., and M.B.J. devised the study. K.P. carried out the fabrication, electrical, and THz-TDS measurements of the devices as well as the FDTD simulations. J.B. performed 
optical pump terahertz probe experiments and together with P.P. assisted with the THz-TDS measurements. Q.G. grew and characterized the nanowire materials. Y.W. assisted with the simulations. C.D. performed the refractive index and absorption coefficient extraction. Z.Y.L. assisted with the device fabrication. K.P., P.P., and L.F. prepared the manuscript. All authors discussed and commented on the manuscript.

\section{Funding}

We acknowledge the Australian Research Council (ARC) and the Engineering and Physical Sciences Research Council (EPSRC) for financial supports.

\section{Notes}

The authors declare no competing financial interest.

\section{ACKNOWLEDGMENTS}

We thank the Australian National Fabrication Facility (ANFF) ACT node for access to the fabrication facilities used in this work, the National Computational Infrastructure (NCI) for providing the computational resources for this work, and Bandwidth Foundry International Pty Ltd for their product service for this work. We appreciate Dennis Gibson from ANU RSPE Electronics Unit for his assistance with the sample holder design and modification, and we are also grateful to Fouad Karouta (ANFF), Kaushal Vora (ANFF), and Marco Zerbini (ENEA Frascati) for useful discussions.

\section{REFERENCES}

(1) Hangyo, M.; Tani, M.; Nagashima, T. Int. J. Infrared Millimeter Waves 2005, 26 (12), 1661-1690.

(2) Nuss, M.; Orenstein, J., Terahertz time-domain spectroscopy. In Millimeter and Submillimeter Wave Spectroscopy of Solids; Grüner, G., Ed.; Springer: Berlin, 1998; Vol. 74, pp 7-50.

(3) Ouchi, T.; Kajiki, K.; Koizumi, T.; Itsuji, T.; Koyama, Y.; Sekiguchi, R.; Kubota, O.; Kawase, K. J. Infrared, Millimeter, Terahertz Waves 2014, 35 (1), 118-130.

(4) Tonouchi, M. Nat. Photonics 2007, 1 (2), 97-105.

(5) Oh, S. J.; Kim, S. H.; Jeong, K.; Park, Y.; Huh, Y. M.; Son, J. H.; Suh, J. S. Opt. Express 2013, 21 (18), 21299-21305.

(6) Auston, D. H. Appl. Phys. Lett. 1975, 26 (3), 101-103.

(7) Beard, M. C.; Turner, G. M.; Schmuttenmaer, C. A. J. Appl. Phys. 2001, 90 (12), 5915-5923.

(8) Doany, F. E.; Grischkowsky, D.; Chi, C. C. Appl. Phys. Lett. 1987, 50 (8), 460-462.

(9) Castro-Camus, E.; Lloyd-Hughes, J.; Fu, L.; Tan, H. H.; Jagadish, C.; Johnston, M. B. Opt. Express 2007, 15 (11), 7047-7057.

(10) Azad, A. K.; Prasankumar, R. P.; Talbayev, D.; Taylor, A. J.; Averitt, R. D.; Zide, J. M. O.; Lu, H.; Gossard, A. C.; O'Hara, J. F. Appl. Phys. Lett. 2008, 93 (12), 121108.

(11) O’Hara, J. F.; Zide, J. M. O.; Gossard, A. C.; Taylor, A. J.; Averitt, R. D. Appl. Phys. Lett. 2006, 88 (25), 251119.

(12) Wu, Q.; Zhang, X. C. Appl. Phys. Lett. 1995, 67 (24), 3523-3525.

(13) Castro-Camus, E.; Fu, L.; Lloyd-Hughes, J.; Tan, H. H.; Jagadish, C.; Johnston, M. B. J. Appl. Phys. 2008, 104 (5), 053113-053119.

(14) Gupta, S.; Whitaker, J. F.; Mourou, G. A. IEEE J. Quantum Electron. 1992, 28 (10), 2464-2472.

(15) Liu, T.-A.; Tani, M.; Nakajima, M.; Hangyo, M.; Pan, C.-L. Appl. Phys. Lett. 2003, 83 (7), 1322-1324.

(16) Vitiello, M. S.; Coquillat, D.; Viti, L.; Ercolani, D.; Teppe, F.; Pitanti, A.; Beltram, F.; Sorba, L.; Knap, W.; Tredicucci, A. Nano Lett. 2012, 12 (1), 96-101.

(17) Romeo, L.; Coquillat, D.; Pea, M.; Ercolani, D.; Beltram, F.; Sorba, L.; Knap, W.; Tredicucci, A.; Vitiello, M. S. Nanotechnology 2013, 24 (21), 214005-214012.

(18) Peng, K.; Parkinson, P.; Fu, L.; Gao, Q.; Jiang, N.; Guo, Y. N.; Wang, F.; Joyce, H. J.; Boland, J. L.; Tan, H. H.; Jagadish, C.; Johnston, M. B. Nano Lett. 2015, 15 (1), 206-210.
(19) Joyce, H. J.; Docherty, C. J.; Gao, Q.; Tan, H. H.; Jagadish, C.; Lloyd-Hughes, J.; Herz, L. M.; Johnston, M. B. Nanotechnology 2013, 24 (21), 214006-214012.

(20) Parkinson, P.; Lloyd-Hughes, J.; Gao, Q.; Tan, H. H.; Jagadish, C.; Johnston, M. B.; Herz, L. M. Nano Lett. 2007, 7 (7), 2162-2165.

(21) Joyce, H. J.; Gao, Q.; Tan, H. H.; Jagadish, C.; Kim, Y.; Zhang, X.; Guo, Y. N.; Zou, J. Nano Lett. 2007, 7 (4), 921-926.

(22) Kawano, Y.; Ishibashi, K. Nat. Photonics 2008, 2 (10), 618-621.

(23) Wood, C.; Cunningham, J.; Hunter, I. C.; Tosch, P.; Linfield, E. H.; Davies, A. G. Int. J. Infrared Millimeter Waves 2007, 27 (4), 557-569.

(24) Cunningham, J.; Byrne, M. B.; Wood, C. D.; Dazhang, L. Electron. Lett. 2010, 46 (26), S34-S37.

(25) Vitiello, M. S.; Viti, L.; Coquillat, D.; Knap, W.; Ercolani, D.; Sorba, L. APL Mater. 2015, 3 (2), 026104.

(26) Dai, X.; Zhang, S.; Wang, Z.; Adamo, G.; Liu, H.; Huang, Y.; Couteau, C.; Soci, C. Nano Lett. 2014, 14 (5), 2688-2693.

(27) Gao, Q.; Saxena, D.; Wang, F.; Fu, L.; Mokkapati, S.; Guo, Y. A.; Li, L.; Wong-Leung, J.; Caroff, P.; Tan, H. H.; Jagadish, C. Nano Lett. 2014, 14 (9), 5206-5211.

(28) Maharjan, A.; Pemasiri, K.; Kumar, P.; Wade, A.; Smith, L. M.; Jackson, H. E.; Yarrison-Rice, J. M.; Kogan, A.; Paiman, S.; Gao, Q.; Tan, H. H.; Jagadish, C. Appl. Phys. Lett. 2009, 94 (19), 193115.

(29) Jiang, N.; Gao, Q.; Parkinson, P.; Wong-Leung, J.; Mokkapati, S.; Breuer, S.; Tan, H. H.; Zheng, C. L.; Etheridge, J.; Jagadish, C. Nano Lett. 2013, 13 (11), 5135-5140.

(30) Joyce, H. J.; Wong-Leung, J.; Yong, C. K.; Docherty, C. J.; Paiman, S.; Gao, Q.; Tan, H. H.; Jagadish, C.; Lloyd-Hughes, J.; Herz, L. M.; Johnston, M. B. Nano Lett. 2012, 12 (10), 5325-5330.

(31) Jiang, N.; Parkinson, P.; Gao, Q.; Breuer, S.; Tan, H. H.; WongLeung, J.; Jagadish, C. Appl. Phys. Lett. 2012, 101 (2), 023111-023114.

(32) Kelrich, A.; Dubrovskii, V. G.; Calahorra, Y.; Cohen, S.; Ritter, D. Nanotechnology 2015, 26 (8), 085303.

(33) Ishizaka, F.; Hiraya, Y.; Tomioka, K.; Fukui, T. J. Cryst. Growth 2015, 411, 71-75.

(34) De Luca, M.; Zilli, A.; Fonseka, H. A.; Mokkapati, S.; Miriametro, A.; Tan, H. H.; Smith, L. M.; Jagadish, C.; Capizzi, M.; Polimeni, A. Nano Lett. 2015, 15 (2), 998-1005.

(35) Wallentin, J.; Anttu, N.; Asoli, D.; Huffman, M.; Aberg, I.; Magnusson, M. H.; Siefer, G.; Fuss-Kailuweit, P.; Dimroth, F.; Witzigmann, B.; Xu, H. Q.; Samuelson, L.; Deppert, K.; Borgstrom, M. T. Science 2013, 339 (6123), 1057-1060.

(36) Wang, F.; Gao, Q.; Peng, K.; Li, Z.; Li, Z. Y.; Guo, Y. N.; Fu, L.; Smith, L. M.; Tan, H. H.; Jagadish, C. Nano Lett. 2015, 15 (5), 30173023.

(37) Yang, Y. P.; Singh, R.; Zhang, W. L. Opt. Lett. 2011, 36 (15), 2901-2903.

(38) Kasai, S.; Tanabashi, A.; Kajiki, K.; Itsuji, T.; Kurosaka, R.; Yoneyama, H.; Yamashita, M.; Ito, H.; Ouchi, T. Appl. Phys. Express 2009, 2 (6), 062401.

(39) Lloyd-Hughes, J.; Fu, L.; Castro-Camus, E.; Merchant, S.; Tan, H. H.; Jagadish, C.; Johnston, M. B. Conference Digest of the 2006 Joint 31st International Conference on Infrared and Millimeter Waves and 14th International Conference on Terahertz Electronics, 2006; pp 149-149.

(40) Boland, J. L.; Conesa-Boj, S.; Parkinson, P.; Tütüncüoglu, G.; Matteini, F.; Rüffer, D.; Casadei, A.; Amaduzzi, F.; Jabeen, F.; Davies, C. L.; Joyce, H. J.; Herz, L. M.; Fontcuberta i Morral, A.; Johnston, M. B. Nano Lett. 2015, 15 (2), 1336-1342.

(41) Duvillaret, L.; Garet, F.; Coutaz, J. L. IEEE J. Sel. Top. Quantum Electron. 1996, 2 (3), 739-746.

(42) Hussain, B.; Nawaz, M.; Ahmed, M.; Raja, M. Y. A. Laser Phys. Lett. 2013, 10 (5), 055301.

(43) Cordes, A. H.; Thomas, D. H.; von der Weid, J. P. Opt. Lett. 2013, 38 (19), 3704-3707.

(44) Hattori, T.; Kumon, H.; Tamazumi, H. 35th International Conference on Infrared, Millimeter and Terahertz Waves (Irmmw-Thz 2010), 2010; pp 1-2.

(45) Yano, R.; Gotoh, H.; Hirayama, Y.; Miyashita, S.; Kadoya, Y.; Hattori, T. J. Appl. Phys. 2005, 97 (10), 103103-103108. 
(46) Nguyen, T. K.; Ho, T. A.; Han, H.; Park, I. J. Infrared, Millimeter, Terahertz Waves 2012, 33 (11), 1123-1137. 